\title{
¿EXISTE UNA VENTAJA DE SALARIO PARA LOS HABITANTES DE LA REGIÓN DE MAGALLANES? ANÁLISIS A PARTIR DE UN ENFOQUE POR GÉNERO
}

\author{
LUZ MARÍA FERRADA* \& VIRGNIA MONTAÑA*
}

\begin{abstract}
RESUMEN
La región de Magallanes es definida Zona Extrema, de manera tal que sus habitantes puedan acceder a mejores condiciones de desarrollo, dado que éstas normalmente surgen y se quedan en el centro geográfico de Chile. Así, en este territorio los salarios en promedio son mayores a los del país aún cuando siguen siendo menores que en la región Metropolitana. En este trabajo se analiza la estructura productiva, ocupacional y los ingresos laborales de ambas regiones. Se estiman funciones salariales de hombres y mujeres, para luego calcular brechas regionales de salarios por género. Las principales conclusiones son que en Magallanes hay una menor dotación de factores, lo que explicaría los bajos salarios de las mujeres, sin embargo, en varones la brecha se anula. Además, se estima que el rendimiento de los factores en mujeres de Magallanes también es inferior, lo que probablemente ocurre porque laboran en sectores con menor dinamismo relativo.
\end{abstract}

PALABRAS CLAVE: disparidades regionales, economía regional, salarios, género.

Clasificación JEL: Z0, R0, J3.

\section{ARE WAGE LEVELS ADVANTAGEOUS FOR THE INHABITANTS OF THE MAGALLANES REGION? A GENDER APPROACH ANALYSIS}

\begin{abstract}
The Magallanes region has been designated "Extreme Zone" status, ensuring residents have access to better development conditions which generally emerge and remain in the geographical centre of Chile. Thus, in this territory, average wages are higher than the rest of the country, although still below those of the Metropolitan region. This study analyzes the production and occupational structure, as well as earned income of both regions. The salary functions of men and women are estimated, in order to calculate regional wage gaps according to gender. The main conclusions are the existence of a lower

Universidad de Los Lagos, Departamento de Ciencias Administrativas y Económicas, Núcleo de Economía Regional, Avda. Fuchslocher 1305, Osorno, código postal 5290000, lferrada@ulagos.cl

" Universidad de Los Lagos, Departamento de Ciencias Administrativas y Económicas, Núcleo de Economía Regional, Avda. Fuchslocher 1305, Osorno, código postal 5290000, vmontana@ulagos.cl

Este artículo forma parte del estudio "Oferta y Disparidades Laborales en Las Regiones de Chile". Proyecto R09-11, financiado por la Dirección de Investigación de la Universidad de Los Lagos.
\end{abstract}


factor endowment in Magallanes, which would account for lower women's salaries. Nevertheless, this gap is not present in the case of men's wages; furthermore, women`s factor performance in Magallanes is also estimated to be inferior, which probably occurs because they tend to work in sectors with a lower relative dynamism.

KEY WORDS: regional disparities, regional economy, wages, gender.

Classification JEL: ZO, RO, J3.

\section{INTRODUCCIÓN}

La economía chilena registra desde la década de 1990 un crecimiento sostenido del PIB, pero con altas tasas de disparidades y concentración económica espacial en torno principalmente a la región Metropolitana, aun cuando estos antecedentes se han planteado con preocupación en la agenda de la política económica de todo el período.

Un caso especial de intervención pública en Chile han sido las políticas en favor de las regiones localizadas en el extremo norte y sur. Estas regiones tienen características que las configuran como regiones aisladas con dificultades para comunicarse $e$ integrarse con resto del territorio nacional. Por su ubicación geográfica son además, regiones fronterizas; Así, los habitantes, tanto del extremo norte como sur, se encuentran más cercanos a territorios de otros países: esto es Perú y Argentina, respectivamente, ya sea por distancia o por fricciones naturales del espacio. De esta forma, el desplazamiento entre Arica e Iquique (ciudades que concentran la población de las regiones del extremo norte de Chile) implica recorrer una distancia de 302 kilómetros, no obstante entre Arica y Tacna (ciudad adyacente ubicada en Perú) hay sólo 45 Kilómetros. En el sur desde Punta Arenas a Coyhaique (ciudades con mayor población ubicadas en el extremo sur), median 1.447 kilómetros de distancia, sin embargo, entre Punta Arena y Río Gallegos (ciudad adyacente ubicada en Argentina) hay sólo 256 km (Fig. 1 de Anexos). Esto es sólo considerando la perspectiva de distancia, puesto que en dichos territorios existen adicionalmente otras fricciones en el espacio geográfico que complican las comunicaciones y el desplazamientos dentro del país. Todo ello repercute, en que esta población tenga que enfrentar dificultades como mayor costo de vida y menor acceso a ciertos bienes y servicios.

Basado en ello, el Estado caracterizó a Magallanes como Zona Extrema, lo que ha implicado otorgar ciertas ventajas a favor de sus habitantes, dos de ellas han sido: la creación de zonas francas y la implementación de asignaciones especiales para los sueldos de empleados públicos. Estas condiciones especiales y otras características de su economía, explican que el salario medio en la región de Magallanes sea mayor al promedio del país, aunque inferior al de la región Metropolitana, siendo mayor la diferencias en las mujeres.

Parece interesante entonces analizar las diferencias salariales entre las regiones de Magallanes y Metropolitana, esto constituye el propósito del estudio. Los objetivos específicos son: (1) analizar la estructura salarial de hombres y mujeres de ambas regiones y (2) explicar las diferencias salariales entre ambas regiones. Las hipótesis nulas que se investigan son:

$\mathrm{H}_{01}$ : Las diferencias regionales de salario se explican tanto por dotación de factores como por rendimiento de ellos.

$\mathrm{H}_{02}$ : La estructura de las brechas regionales de salarios de las mujeres es igual a la de los varones.

En el trabajo se analiza la distribución del PIB y la ocupación por rama de actividad económica, luego se estiman funciones salariales regionales, por género. Con ello, se calculan brechas regionales de salarios, de hombres y mujeres en forma separada, para finalmente verificar cuánto de dicha diferencia se debe a que los habitantes de una determinada región cuentan con mayor o menor cantidad de características valoradas por el mercado laboral.

En adelante el artículo se estructura de la siguiente forma: en primer lugar se exponen antecedentes bibliográficos de modo de colocar en contexto la problemática investigada, luego se detalla la metodología y datos a utilizar, para posteriormente presentar los resultados, y finalmente, concluir con relación a la hipótesis y objetivos de la investigación. 


\section{ANTECEDENTES}

Con el propósito de comprender las diferencias en cuanto a salarios entre ambas regiones se analiza literatura en tres aspectos que se consideran importantes para el caso de estudio: Primero se revisa el concepto Zona Extrema usado en Chile, para así entender la lógica que tuvo su aplicación en la región de Magallanes y los efectos esperados de ella en los salarios. Sin embargo, los resultados no han sido del todo satisfactorio, probablemente ello sea consecuencia de la tendencia centralista que ha seguido la política pública chilena, esto es lo que argumenta en el punto siguiente. Finalmente, se resumen antecedentes de la economía regional de las últimas décadas, destacando los efectos de la aplicación de las políticas compensatorias implementadas en la región de Magallanes.

\section{Magallanes como Zona Extrema}

Siendo Magallanes una región aislada y fronteriza, el Estado de Chile la ha definido como Zona Extrema (ZE) instalando distintas leyes especiales que permitan a sus habitantes facilitar el acceso a las condiciones de desarrollo del país.

Se entiende por territorios especialmente aislados a aquellos territorios que geográficamente están aislados, con dificultades para comunicarse e integrarse al resto del país $y$ con escasa y dispersa población, escaso acceso a los servicios públicos y se asocian a las zonas fronterizas ubicadas a ambos extremos del país (CIDEZE-SUBDERE, 2007:22). En esta categoría se encuentra la región de Magallanes de acuerdo al Comité para el Desarrollo de Zonas Extremas y Especiales (CIDEZE) ${ }^{1}$.

Sin embargo, en la definición de las ZE no sólo han influido factores geográficos, sino también "históricos y geopolíticos" (Banco Mundial, 2005:3). Además, en estas ZE y fronterizas, se generan relaciones complejas con las zonas aledañas externas (Slater, 2013:13), así, los habitantes de la región de Magallanes (ya sea por las distancias o fricciones del espacio geográfico) tienen un alto nivel de vinculaciones con el territorio

1 Organismo consultivo de carácter permanente en materia de estudios y formulación de políticas, planes y argentino. Es por ello, que el Estado ha planteado la necesidad de consolidar la soberanía en estas zonas.

Otros elementos para que estas regiones sean calificadas como extremas, son el alto costo de vida y la lejanía que tienen a los polos de comercio nacional e internacional. Según el Banco Mundial si bien inicialmente no se definieron estrategias explícitas para alcanzar estos objetivos, se puede argumentar que el camino elegido en la práctica ha sido el de fomentar el desarrollo económico de las ZE (Banco Mundial, 2005:7).

A partir del año 2007 se plantea superar el concepto de Zona Extrema Especial por el de Territorios Especiales Aislados, elaborando en el año 2008 un Plan de Incentivos Especiales para Zonas Extremas y una Política Nacional para el Desarrollo de Localidades Aisladas (BCN, 2011:2).

No obstante, en los hechos, la visión del Estado de Chile respecto de sus ZE se contradice con el ejercicio de articulación de políticas públicas en función de ellas (Correa \& Soza, 2012), en realidad éste sigue tendiendo a la concentración de la economía en torno a la región Metropolitana, como históricamente lo ha sido.

\section{Concentración del crecimiento}

Si bien, la economía chilena ha registrado en las últimas décadas un crecimiento sostenido, este se ha generado en un territorio económicamente muy heterogéneo, que produce altos índices de desigualdad y concentración espacial.

La literatura indica que el crecimiento en condiciones de la concentración espacial es ineficiente después de cierto umbral de PIB per cápita, y se ha demostrado que en Chile este limite se ha superado; por lo tanto, las políticas de crecimiento que tienden a la concentración generan efectos negativos (Atienza \& Aroca, 2012). Así, el problemas es complejo al constatar, que el incremento de las diferencias entre los salarios regionales y de la región Metropolitana, es producto no sólo de los mecanismos de mercado sino también de la política pública aplicada (Aroca, 2009).

programas tendientes al desarrollo de estas zonas 
Las regiones observan una fuerte especialización productiva, en particular las intensivas en explotación de recursos naturales $y$ se ven expuestas a riesgos de sustentabilidad económica y social, pues dependen fuertemente de los ciclos de la demanda internacional (Atienza \& Aroca, 2012). De esta forma, se configura un territorio muy heterogéneo: con alta especialización en actividades mineras en el norte y la región de Magallanes, en el sector silvoagropecuario y pesca en el sur, y fuerte concentración de servicios financieros y otros en torno a la región Metropolitana. En estas circunstancias, una decisión política pública centralista pueden afectar gravemente a algunas economías regionales; esto es, una política que favorece al centro pudiese convertirse en un obstáculo para el crecimiento económico de otra región del país. Así, los mecanismos de políticas públicas no contrapesan los efectos de las fuerzas del mercado.

\section{Evidencia en cuanto al efecto de la intervención en Magallanes}

La actividad industrial en Magallanes nace a fines de siglo XIX, producto de la intervención del Estado, en particular con la colonización pastoril de la zona, con la consecuente producción de lana y piel, lo que dinamiza la industria ganadera y alimentaria; también es la época en que se desarrolla la actividad forestal y minera (Martinic, 2001).

Posteriormente, surge la industria de los hidrocarburos, aumentando el dinamismo de la economía regional. Sin embargo, en la segunda mitad del siglo pasado la industria comienza a contraerse. Latorre (1999) para un análisis entre los años 1960 a 1990, encuentra que esta región creció a un ritmo inferior al del país; este menor crecimiento es explicado por un cambio estructural, que tiene origen en la menor participación en el PIB minero, que denota a una región menos especializada en esta actividad que es de muy rápido crecimiento. Estudiando el período 19901997, Rifo (1999) concuerda con lo anterior, aunque encuentra que ésta es la región del país con mayor comportamiento convergente.

El cambio estructural ha permitido el desarrollo de ciertos sectores emergentes, y con ello una región menos especializada; es así como SozaAmigo (2008) observa la configuración de cuatro clúster en la economía de magallánica, uno de ellos lo conforman las actividades encadenadas a la producción minera derivada de los hidrocarburos; otros son: el clúster formado por los sectores productos agrícolas, servicios de hotelería y restaurantes y servicios de transporte marítimo y aéreo; el clúster que incluye al vidrio, minerales no metálicos, hierro, acero y productos metálicos y construcción; y el compuesto por sectores servicios comerciales y de trasporte ferroviario y caminero.

También destaca un emergente sector turismo, así en otro trabajo el mismo autor (SozaAmigo, 2009) indica que este sector en la región es la que aglutina más actividades, esto es, que depende del resto del sistema y este a su vez de él. Además plantea, que las regiones extremas guardan un cierto parecido estructural.

De esta forma, diversos indicadores de la región muestran resultados atractivos, con relación al promedio del país, en particularmente de competitividad regional, distribución de ingreso y

Tabla 1 Indicadores para las regiones Metropolitana y Magallanes.

\begin{tabular}{|l|c|c|c|}
\hline \multicolumn{1}{|c|}{ Región } & Magallanes & Metropolitana & País \\
\hline Índice de Competitividad (2008) & 359,64 & 358,05 & 277,94 \\
\hline Índice de GINI, sobre ingresos autónomos 2011(2) $^{(2)}$ & 0,51 & 0,53 & 0,54 \\
\hline Porcentaje pobreza 2011(2) $^{(2)}$ & 5,3 & 10,5 & 14,4 \\
\hline Promedio de escolaridad 2011(2) & 10,8 & 11,2 & 10,5 \\
\hline Índice de Desarrollo Humano (IDH) 2003 ${ }^{(3)}$ & 0,788 & 0,812 & 0,773 \\
\hline PIB regionalizado en millones de pesos encadenados (2009)(4) & 755,381 & 40.416 .229 & 85.800 .078 \\
\hline PIB per cápita en pesos al mes (2009)4 & 398,128 & 494,234 & 422,356 \\
\hline
\end{tabular}

Fuente: (1) SUBDERE. (2009); (2) Encuesta CASEN, 2011; (3) PNUD, 2004; (4) Banco Central e INE. 
Tabla 2 Ingresos laborales de las regiones Metropolitana, Magallanes y país por sexo, en pesos de cada año.

\begin{tabular}{|c|c|c|c|c|c|c|c|c|c|}
\hline \multicolumn{9}{|c|}{ Total } & \multicolumn{9}{c|}{ Mombre } & \multicolumn{5}{c|}{ Mujer } \\
Región & Magallanes & Metropolitana & País & Magallanes & Metropolitana & País & Magallanes & Metropolitana & País \\
\hline 1990 & 85,656 & 94,01 & 82,643 & 94,797 & 108,132 & 92,566 & 66,142 & 68,451 & 60,835 \\
\hline 1992 & 139,276 & 155,47 & 126,594 & 154,996 & 177,475 & 140,81 & 101,158 & 113,32 & 95,507 \\
\hline 1994 & 170,674 & 220,665 & 171,852 & 191,847 & 249,569 & 188,71 & 127,476 & 168,803 & 136,57 \\
\hline 1996 & 221,67 & 271,752 & 216,891 & 265,57 & 310,607 & 240,51 & 145,152 & 205,873 & 169,743 \\
\hline 1998 & 269,232 & 326,035 & 265,013 & 320,477 & 374,483 & 294,89 & 172,314 & 247,68 & 209,229 \\
\hline 2000 & 388,661 & 342,598 & 287,338 & 443,366 & 403,189 & 329,19 & 303,495 & 247,018 & 211,792 \\
\hline 2003 & 330,742 & 392,996 & 307,749 & 380,005 & 469,621 & 349,09 & 230,696 & 275,199 & 235,188 \\
\hline 2006 & 331,248 & 381,092 & 309,501 & 361,199 & 441,044 & 347,14 & 284,085 & 295,23 & 248,582 \\
\hline 2009 & 421,217 & 522,297 & 412,768 & 518,572 & 620,734 & 472,41 & 253,988 & 383,793 & 319,2 \\
\hline
\end{tabular}

Fuente: Resultados Encuesta CASEN de cada año.

tasas de pobreza. Como se observa (Tabla 1), en éstos se obtienen resultados superiores inclusive a los de la región Metropolitana.

Una primera interpretación de los resultados al compararlos con los del país, es que las políticas implementadas, luego de configurar a la región como ZE, han tenido efectos relevantes para la economía magallánica; no obstante, hay estudios que concluyen que dichas políticas de intervención no han influido claramente en el desempeño regional. Concretamente, se indica que si en Magallanes no se hubiesen aplicado políticas compensatorias la participación en la población nacional en el período 1970-2002 se hubiese mantenido; además, se estima (para período 19862001) que si se hubiese crecido como el país, con la estructura inicial (antes del cambio estructural), el resultado hubiese sido mayor al crecimiento efectivamente obtenido (Banco Mundial, 2005).

Como se observa en la misma tabla, la región de Magallanes tiene menor PIB per cápita e IDH que la Metropolitana; pero además, la región ha bajando su participación en el PIB nacional en el largo plazo, del 3\% registrado en el año 1985 se cae al 0,7\% en 2011 (Banco Central). Ahora bien, en ingresos laborales la región de Magallanes obtiene siempre un salario medio inferior al de la Metropolitana, en particular en mujeres, como se observa en la Tabla 2.

Rifo (1999) indica que las desigualdades regionales se encuentran determinadas principalmente por las diferencias en productividad, la que pudiese ser explicada por las distintas dotaciones espaciales de factores.

$\mathrm{Al}$ respecto, un problema ampliamente investigado en Chile es la diferencia de ingresos laborales entre hombres y mujeres. Sin embargo, poco se ha avanzado en el estudio de brechas regionales. Ñopo (2006) estima brechas de género, por región, y concluye que no es claro que la brecha salarial no explicada en Santiago sea mayor al resto del país, sin embargo Bravo et al (2008) avanza al distinguir entre tres zonas norte, centro, sur y Santiago, y obtiene que si la residencia es Santiago la brecha aumenta, en particular en aquellos con mayores niveles educativos. Sin embargo, los niveles educativos en Chile han aumentado fuertemente en todas las regiones; pero este incremento no ha logrado reducir las disparidades salariales, probablemente porque los mayores rendimientos educacionales se han trasladado a niveles cada vez más altos, desde educación media a la educación universitaria (Beyer \& Le Foulon, 2002).

\section{METODOLOGÍA Y DATOS}

Para verificar las hipótesis y desarrollar los objetivos del estudio se abordan tres aspectos: (1) la estructura productiva y ocupacional de la región de Magallanes que se compara con los antecedentes de la región Metropolitana; (2) la estructura salarial de ambas regiones; y (3) la estimación de brechas regionales de los salarios por género.

El trabajo se realiza con los siguientes antecedentes y fuentes: 
- Producto interno bruto (PIB) regionalizado, publicados por el Banco Central de Chile.

-Ocupación por región, de la Nueva Encuesta de Empleo (NENE) y publicado por el Instituto Nacional de Estadísticas (INE).

-Ingresos, y variables explicativas del salario, obtenidos de la base de datos de Encuesta de Caracterización Socioeconómica Nacional (CASEN), publicada por el Ministerio de Desarrollo Social.

Los datos corresponden al año 2009, y para el caso de la NENE (encuesta presentada en trimestres móviles) se escoge el periodo que coincide con el trabajo en terreno de la encuesta CASEN en dicho año, esto es el trimestre móvil Octubre-Diciembre.

A continuación se expone la forma de trabajar cada uno de los aspectos mencionados.

\section{Estructura productiva y ocupacional}

En este caso se estudia la distribución de PIB y ocupación en ambas regiones. Para ello se calculan tasas de participación sectorial, comparando la importancia que tiene cada sector entre las regiones. En el caso de la ocupación se analizan además, las diferencias regionales por sexo

Análisis de salarios

Se realizan dos análisis, primero descriptivo y luego explicativo. Con los antecedentes de la base de datos de encuesta CASEN se analiza la estructura salarial por región y sexo. Se seleccionan los antecedentes de aquellos informantes que responden a la pregunta sobre su ingreso laboral y el número de horas que trabajan a la semana, para luego estudiar el salario por hora partir de dicha variable. Además, conforme a las estadísticas laborales chilenas se seleccionan a los informantes que indican tener, al menos 15 años de edad en las mujeres hasta 60 años y en los varones hasta 65 años, esto en base a la edad de jubilación definida en Chile.

En la segunda etapa, se busca conocer la función salarial por región para comparar entre ellas cómo ciertas variables explicativas del salario en ambas regiones influyen en los salarios regionales de hombres y mujeres. En total se realizan cuatro estimaciones, para hombres de la región de Magallanes y Metropolitana, y para mujeres de las dos regiones. Cada ecuación se modela como:

$$
\text { h } w_{i}=X_{i} \beta_{i}+\mu_{i}
$$

Siendo Inw el logaritmo natural del salario, $i$ un subíndice que representa las observaciones de 1 a $\mathrm{N}$, que corresponden a las personas en condición laboral ocupadas, $X$ es una matriz de variables independientes, $\beta$ un vector columna de coeficientes a estimar, y $\mu$ los términos de perturbaciones.

Como es sabido, en este tipo de trabajos se tiene el problema de sesgo de selección, dado que se usan muestras no seleccionadas aleatoriamente, una muestra censurada, pues se tienen estimaciones de salarios sólo para una parte de la muestra, esto es, las personas que se encuentran en condición de actividad ocupadas. Para Chile el problema es mayor en las mujeres, las que tienen participación laboral muy baja, en la muestra utilizada la tasa de ocupación laboral de la población femenina entre 15 y 60 años es de un 43,3\%, en cambio en los varones es de 69,90\% (Casen, 2009). Ello puede causar distorsiones en la estimación de la ecuación salarial. Para resolver este problema se utiliza el método propuesto por Heckman (1979) (ver anexo 2).

\section{Brechas salariales}

Como se ha indicado, el objetivo del trabajo es indagar acerca las diferencias salariales entre las regiones de Magallanes y Metropolitana, mediante un análisis por género, y se quiere verificar dos hipótesis: (1) que las brechas regionales se explican tanto por dotación de factores como por rendimiento y (2) la estructura de las brechas regionales de las mujeres es igual a la de los varones. Para ello se utiliza la metodología propuesta por Oaxaca (1973), esto es, a partir de las estimaciones realizadas conforme a la ecuación (1), se estiman las brechas, las que se descomponen de la siguiente forma:

$$
\mathrm{h} \bar{w}_{r}-\mathrm{h} \bar{w}_{r^{*}}=\underbrace{\left(X_{r}-X_{r^{*}}\right) \hat{\beta}}+\underbrace{X_{r^{*}}\left(\hat{\beta}_{r}-\hat{\beta}_{r^{*}}\right)}
$$

Siendo la media del logaritmo natural del salario, el subíndice $r$ representa la región de 


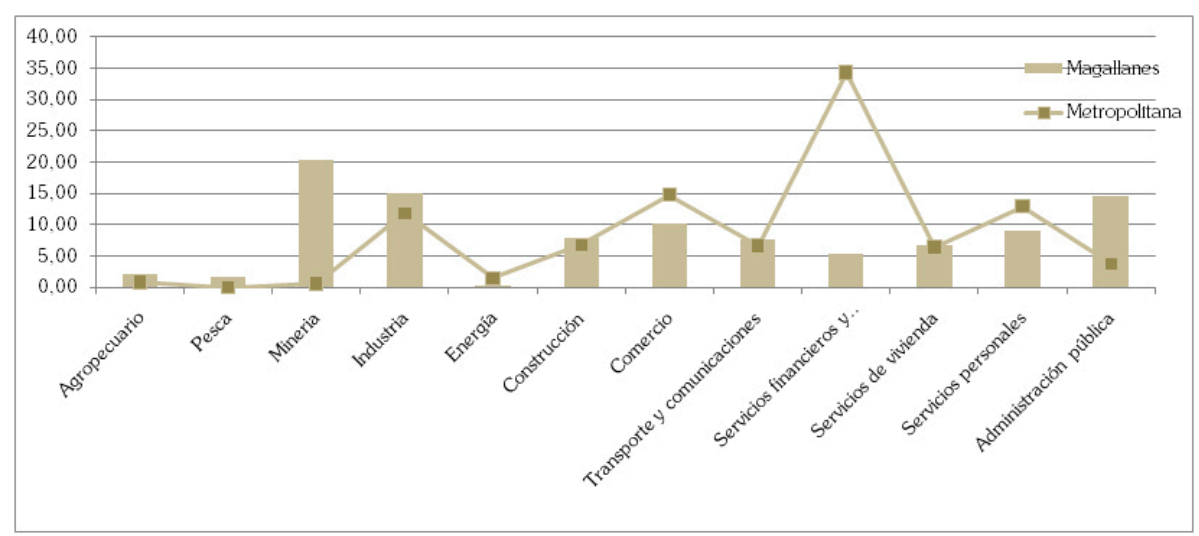

Fig. 1. Distribución porcentual del PIB regional, de las regiones Magallanes y Metropolitana.

Magallanes y $r^{*}$ la región Metropolitana. Siguiendo a Oaxaca (1973), la diferencia salarial entre ambas regiones, que queda en el lado derecho de la ecuación, se descompone en dos partes:

a) Diferencias asociadas a capital humano y otras características de los individuos;

b) Disparidades de salarios porque los rendimientos de determinados factores son distintos entre las regiones $\left(\hat{\beta}_{r}-\hat{\beta}_{r^{*}}\right)$, lo que pudiese ser atribuible a características propias de la región que se esté estudiando.

\section{Variables}

Para el estudio, la variable salarios es el ingreso de la ocupación principal mensual que percibe el individuo dividido por el número de horas que trabaja en esa ocupación a la semana, y para las estimaciones la variable dependiente es el logaritmo natural de dicha variables (lnw); el salario y las horas se obtienen de sus respuestas a las preguntas sobre "ingreso de la ocupación principal" y "horas que efectivamente trabaja a la semana en esa ocupación principal", definidas en la encuesta CASEN 2009.

Las variables explicativas se consideran exógenas, estas son: experiencia potencial ${ }^{2}$ (expr), experiencia potencial al cuadrado (exper2), número de personas en su hogar (personas), si su residencia es en el sector urbano o rural (urbano), si está o no contratado (contrato), si el sector productivo en que trabaja es el primario (primario), secundario (secundario) o terciario (terciario), si trabaja en el sector privado o público (público) y si su último curso en educación formal es educación primaria (edu1), secundaria (edu2) o superior (edu3).

\section{RESULTADOS}

Los resultados de la investigación se exponen siguiendo el orden plantado en la metodología: primero se presenta el análisis de la estructura productiva y ocupacional, luego las estimaciones para los salarios; y finalmente, las brechas salariales y su descomposición conforme al desafío planteado al inicio de este estudio.

\section{Estructura productiva y ocupación}

Los sectores productivos de la región de Magallanes con mayor participación en el PIB regional son Minería (20,4\%), Administración Pública (14,5\%) y Manufactura (14,9\%). Particularmente en los dos primeros se produce una mayor diferencia con la región Metropolitana, estos sectores aportan, respectivamente, 19,8 y 10,8 puntos porcentuales más, al PIB de la región de Magallanes que a la Metropolitana, como se observa en la Fig. 1.

A su vez, tres son los sectores que registran menor aporte porcentual al PIB en comparación con la región Metropolitana son: Comercio, Servicios Personales y Servicios Financieros y Empresariales. Destaca este último sector, ya que la diferencia alcanza a 29 puntos porcentuales.

En cuanto a la distribución sectorial de la ocupación, se aprecia (ver Tabla 3) que la demanda laboral de ambas regiones se concentra en Comercio, Administración Pública y Servicios

\footnotetext{
2 Calculado como: edad-años de escolaridad-6.
} 
Tabla 3 Distribución porcentual de la ocupación por sector, para cada región y sexo.

\begin{tabular}{|l|c|c|c|c|c|c|}
\hline & \multicolumn{3}{c}{ Total } & \multicolumn{3}{c}{ Mujeres } \\
\multicolumn{1}{|c|}{ Región } & Magallanes & Metropolitana & Magallanes & Metropolitana & Magallanes & Metropolitana \\
\hline Silvo agropecuario & 2,73 & 2,69 & 0 & 1,22 & 4,4 & 3,68 \\
\hline Minería & 2 & 0 & 0,67 & 0 & 2,81 & 0 \\
\hline Pesca & 1,6 & 0,57 & 0,65 & 0,07 & 2,18 & 0,9 \\
\hline Industria Manufacturera & 6,07 & 13,89 & 4,41 & 9,8 & 7,08 & 16,66 \\
\hline Electricidad, gas y agua & 0,34 & 0,46 & 0 & 0,23 & 0,54 & 0,62 \\
\hline Construcción & 9,34 & 6,96 & 0 & 1,05 & 15,07 & 10,98 \\
\hline Comercio & 19,65 & 22,24 & 23,88 & 23,49 & 17,05 & 21,39 \\
\hline Hoteles y restaurantes & 5,19 & 3,97 & 9,3 & 4,08 & 2,66 & 3,89 \\
\hline Transporte /comunicación & 4,86 & 8,48 & 2,38 & 3,47 & 6,39 & 11,88 \\
\hline Intermediación financiera & 4,7 & 3,42 & 5,97 & 3,88 & 3,92 & 3,1 \\
\hline Actividades inmobiliarias & 5,52 & 9,51 & 5,31 & 8,73 & 5,65 & 10,03 \\
\hline Administración pública & 16,24 & 5,03 & 5,4 & 5,04 & 22,88 & 5,02 \\
\hline Enseñanza & 8,89 & 7,06 & 16,11 & 11,66 & 4,47 & 3,94 \\
\hline Otros servicios personales, sociales & 12,89 & 15,74 & 25,91 & 27,28 & 4,89 & 7,91 \\
\hline Total & 100 & 100 & 100 & 100 & 100 & 100 \\
\hline
\end{tabular}

Fuente: Nueva Encuesta de Empleo Octubre- Diciembre 2009, INE.

Personales. Estos sectores demandan más de la mitad de la fuerza de trabajo ocupada, lo que se da en mayor proporción en Magallanes principalmente por la alta absorción de empleos en el sector público. En los otros sectores productivos, se observa que en Magallanes se demanda un mayor porcentaje en la actividad de Construcción, en cambio en la Región Metropolitana el empleo relativo es más alto en el sector Manufactura y Actividades Inmobiliarias.

En suma, con respecto al PIB se obtiene, que las diferencias estructurales entre las regiones de Magallanes y Metropolitana están principalmente en Minería, Administración Pública y FinancierosEmpresariales, siendo a favor de Magallanes en las dos primeras. En cuanto al empleo la diferencia está en la mayor ocupación que absorbe la Administración Pública y Construcción de Magallanes.

En este contexto y conforme a la preocupación planteada al inicio de este estudio, se analiza la distribución de la demanda laboral por género. Se obtiene, que las mujeres de ambas regiones trabajan principalmente en Servicios Personales y Comunales, Comercio y Enseñanza, donde se encuentra más del $60 \%$ de la ocupación femenina. Sin embargo, se observa que en Magallanes, a diferencia de la región Metropolitana, se ocupan en mayor proporción en Hoteles y Restaurantes, y Enseñanza, probablemente sectores que tienen bajo valor agregado al menos de acuerdo el PIB observado (Fig. 1). En cambio, la ocupación de las mujeres de la región Metropolitana se diferencia por la mayor proporción de ocupadas en Industria y Actividades Inmobiliaria, ambos sectores con una también mayor participación relativa del PIB.

$\mathrm{Al}$ contrario, los varones de Magallanes obtienen una ventaja relativa respecto de la región Metropolitana, en ocupaciones de Administración pública, Construcción, Minería y Pesca, esto es, en sectores de mayor importancia relativa en el PIB regional y con mejor distribución.

\section{Estructura salarial}

Pues bien, de la Tabla 2 se aprecia que existen diferencias de salarios por género entre las regiones. En promedio, el salario de la región Metropolitana es mayor al de Magallanes, tanto para hombres como mujeres, siendo mayor la diferencia en ellas.

Siguiendo la lógica del punto anterior, se calculan los salarios medios de la ocupación principal mensual por hora semanal de los y las trabajadoras de ambas regiones, por sector productivo, y luego se estiman las diferencias regionales por sector, lo que se exponen en la Fig. 2.

Se aprecia, que en general los salarios medios en todos los sectores son mayores en la región 


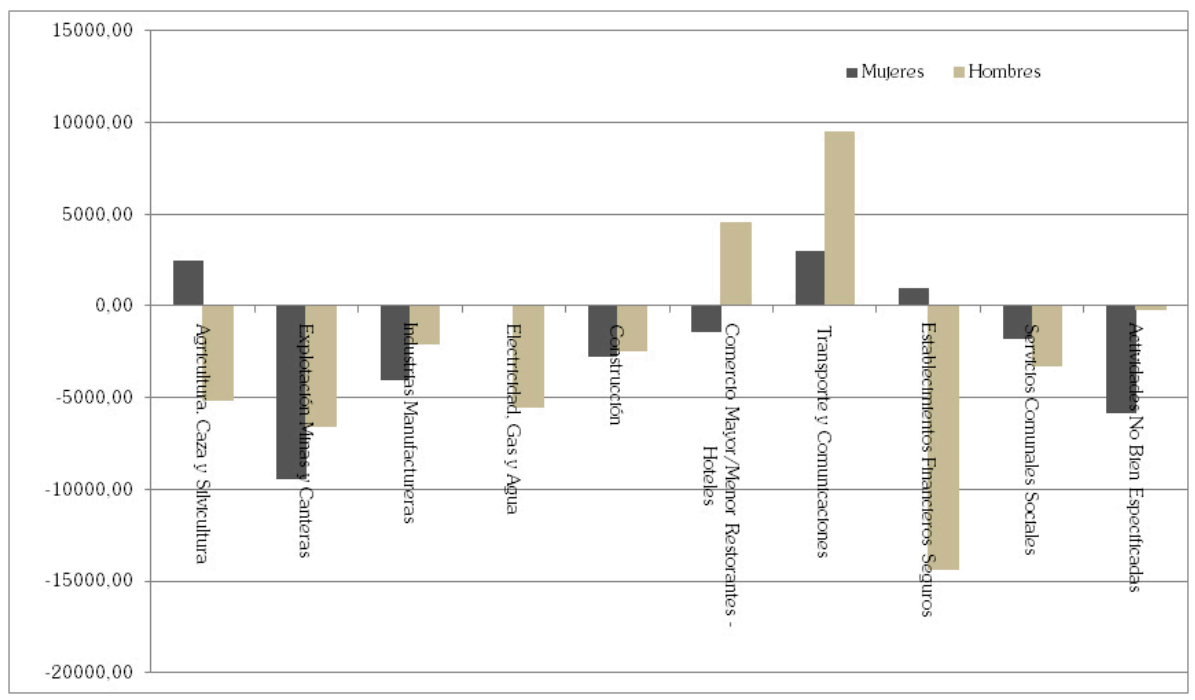

Fig.2. Diferencias de salario medio por hora semanal entre Magallanes y región Metropolitana por sexo y sector.

Metropolitana, salvo en los varones de los sectores Transporte y Comercio, y levemente las mujeres en el sector Agrícola y Financiero.

Para indagar acerca de estas diferencias se estiman cuatro ecuaciones de salarios, considerando como variables explicativas aquellas asociadas al capital humano (experiencia y nivel educativo), asociadas a sectores productivos, condiciones de trabajo (contrato), estructura del hogar (número de personas) y residencia (zona). Antes de analizar los resultados, se estima el salario por hora semanal de trabajo según cada una de las variables mencionadas, lo que se detalla en la Tabla 4.

Se observa que los varones de ambas regiones, que se ubican en zonas urbanas, con contrato, que trabajan en el sector terciario $y$ tienen un nivel educativo superior, obtienen en promedio salarios mayores que los que viven en zonas rurales, sin contrato, trabajan en el sectores primarios o secundarios o tienen niveles educativos inferiores. Sin embargo, hay diferencias en cuanto al sector público/privado, de modo tal que, los trabajadores del sector público de la región Metropolitana obtienen un salario promedio mayor que los del sector privado, en cambio, en Magallanes prácticamente no hay diferencias.

En las mujeres los resultados son más diversos, las variables con mayor consistencia son sector y educación. En ambas regiones, las mujeres que trabajan en el sector público, o tienen nivel educativo superior, obtienen mayores ingresos que las que laboran en el privado, o registran educación menor a la superior.

De la Tabla 4 además se aprecia, que el mayor salario medio por hora semanal que obtienen los habitantes de la región Metropolitana difiere por sexo; así, la disparidad es mayor en las mujeres, se estima una diferencia de $\$ 1.255$ en los varones y de $\$ 2.041$ en las mujeres.

Ahora bien, con los modelos estimados (Tabla 1 de Anexos) se analiza de impacto de cada una de las variables consideradas. Se aprecia que los factores educación, contrato de trabajo, sector productivo y si trabaja en el sector público o privado son, en general, lo que más inciden en el monto del salario, no obstante existen ciertas diferencias por género y región.

En los varones estas variables tienen impactos altos, significativos y aunque el valor del coeficiente pueda ser distinto entre las regiones el signo es el mismo, es decir, tanto para la región Metropolitana como Magallanes se confirma que los que tienen contrato, trabajan en el sector público o poseen mayores niveles educativos, obtienen salarios mayores.

De igual forma, para las mujeres de Magallanes se confirma que una mayor educación y trabajar en el sector público se asocian a mejores salarios, sin embargo, contrario a lo esperado (Tabla 4), el contrato y trabajar en el sector terciario tienen un efecto negativo (Tabla 1 de Anexos) En el mismo sentido, las que tienen educación secundaria reciben menos salario por hora que las con educación primaria o superior. Al contrario, las mujeres de la 
Tabla 4 Salario medio por hora para hombres y mujeres de las regiones de Magallanes y Metropolitana, según distintos factores (en pesos chilenos de 2009).

\begin{tabular}{|l|l|c|c|c|c|}
\hline \multirow{2}{*}{ Variable } & & \multicolumn{2}{|c|}{ Magallanes } & \multicolumn{2}{c|}{ Metropolitana } \\
\hline \multirow{2}{*}{ Zona } & & Hombres & Mujeres & Hombres & Mujeres \\
\hline \multirow{3}{*}{ Contrato } & Rural & 11,095 & 8,944 & 9,69 & 9,824 \\
\cline { 2 - 6 } & Urbano & 13,767 & 8,383 & 15,029 & 10,466 \\
\hline \multirow{3}{*}{ Sector Productivo } & Con Contrato & 9,961 & 6,759 & 11,262 & 9,411 \\
\cline { 2 - 6 } & Sin Contrato & 5,913 & 13,023 & 8,476 & 7,772 \\
\hline \multirow{3}{*}{ Sector } & Primario & 9,344 & 7,92 & 14,64 & 7,941 \\
\hline \multirow{3}{*}{ Nivel Educativo } & Secundario & 9,822 & 5,731 & 12,042 & 10,121 \\
\cline { 2 - 6 } & Terciario & 16,158 & 8,679 & 16,131 & 10,544 \\
\hline & Privado & 13,649 & 8,316 & 14,766 & 9,929 \\
\cline { 2 - 6 } & Público & 13,417 & 8,732 & 15,737 & 14,147 \\
\hline & Básica & 9,625 & 10,443 & 7,319 & 5,862 \\
\cline { 2 - 6 } & Media & 12,126 & 6,748 & 8,678 & 7,07 \\
\cline { 2 - 6 } & Superior & 20,858 & 10,363 & 27,986 & 16,315 \\
\hline
\end{tabular}

Fuente: Base de datos de la Encuesta Casen 2009.

región Metropolitana con contrato, que laboran en el sector servicio o tienen educación secundaria obtienen más salario que las no contratadas, de otros sectores productivos o con menores niveles educativos.

Además, se obtienen diferencias importantes entre hombres y mujeres de la región de Magallanes en cuanto al efecto en el salario de la experiencia y zona de residencia; se aprecia que la experiencia afecta positivamente en los salarios en las mujeres, en cambio en los varones el impacto es muy bajo y negativo; al contrario el trabajar el vivir en zonas urbanas afecta positivamente en los salarios, sin embargo el impacto es mayor en los varones, corroborando los antecedentes de la Tabla 4.

\section{Brechas salariales}

Con las ecuaciones analizadas en el título anterior, y presentadas en la Tabla 1 de los anexos, se estima cuánto sería el ingreso que obtendría, un hombre y una mujer promedio en cada región. Por ejemplo, el modelo estimado permite predecir un salario por hora semanal, para las mujeres de la región Metropolitana de $\$ 6.650$ en promedio, en cambio, para las que residen en Magallanes de $\$ 5.584$; esto es, ambas estimaciones predicen aproximadamente un $60 \%$ del valor observado (Tabla 5). Con base a esta predicción se obtienen brechas salariales entre regiones, para hombres y mujeres.

Es así como, para las mujeres se calcula que las de Magallanes obtienen un $16 \%$ menos de salario que las del centro del país, sin embargo, en los varones la diferencia es positiva, aunque sólo de un $1,2 \%$ (Tabla 5). Por lo tanto, se verifica que existen brechas salariales significativas para el caso de las mujeres de Magallanes.

Ahora bien, las brechas estimadas se pueden explicar porque la región de Magallanes cuenta con menor dotaciones de factores, es decir en promedio,

Tabla 5 Descomposición de Oaxaca por región para mujeres y hombres

\begin{tabular}{|c|c|c|c|c|}
\hline & & Predicción & Diferencia (\$) & Diferencia (\%) \\
\hline \multirow{2}{*}{ Mujeres } & Magallanes & $5.584,3$ & \multirow{2}{*}{$-1.065,7$} & \multirow{2}{*}{$-16,03$} \\
\hline & Metropolitana & $6.650,0$ & & \\
\hline \multirow{2}{*}{ Varones } & Magallanes & $7.462,1$ & \multirow{2}{*}{89,2} & \multirow{2}{*}{1,21} \\
\hline & Metropolitana & $7.373,0$ & & \\
\hline
\end{tabular}

Fuente: En base a los resultados del Anexo 4 y 5. 
Tabla 6. Descomposición de Oaxaca para mujeres y hombres.

\begin{tabular}{|l|c|c|c|}
\hline & Diferencia (\%) & Explicada por dotación & Explicada por rendimiento \\
\hline Mujeres & $-16,03$ & $-8,61 \%$ & $-8,12 \%$ \\
\hline Varones & 1,21 & $-2,33 \%$ & $3,63 \%$ \\
\hline
\end{tabular}

Fuente: En base a los resultados del Anexo.

las trabajadoras tienen menos experiencia, cualificación, entre otras características, que las del centro. Para verificar esta hipótesis, las brechas estimadas se descomponen en diferencial por dotación de factores y por rendimientos, según lo explicado en el apartado anterior. Los resultados se presentan la Tabla 6.

Se observa que la mitad de la brecha salarial de las mujeres (que es $16 \%$ ) se explica por diferencias en dotación de factores. Ello quiere decir que si el promedio de las dotaciones fuesen iguales en ambas regiones la brecha se reduciría a la mitad, esta conclusión es relevante porque podría tener consecuencias para la política pública. La otra mitad de la brecha que, se debe a diferencias en rendimiento de los factores; es decir, aún cuando las mujeres tengan la misma dotación de factores de igual manera se produciría una diferencia de un $8 \%$ en el salario medio por hora semanal y está explicado porque en la región Metropolitana se premia más el contar con dichos factores. En este caso probablemente ello se relaciona las diferentes estructuras productivas que tienen ambas regiones y con la mayor productividad relativa de los sectores en la región Metropolitana.

Para los varones los resultados son muy diferentes. No hay brecha salarial porque, aunque los trabajadores de Magallanes observan una menor dotación de factores (como niveles de educación) se calculó incluso una pequeña brecha positiva (del 1,2\%) que no es significativa estadísticamente (Tabla 3 de Anexos). Es decir, si su dotación aumentase a niveles de los trabajadores de la región Metropolitana su salario en promedio sería superior en un 2,3\% (Tabla 6), y mayor aún si se considera que en los de Magallanes dichos factores se premian mejor, en la media en un 3,6\% más que en la región Metropolitana.

\section{DISCUSIÓN Y CONCLUSIONES}

Al inicio del trabajo se constató que en promedio el salario medio en la región de Magallanes es mayor al promedio del país, pero no superior al de la región Metropolitana. Posteriormente, se observa que la diferencia es mayor en las mujeres. Pues bien, a través de la estimación de ecuaciones de salario se constata el resultado para las mujeres, pero no para los varones.

Para intentar explicar estas diferencias, se ha descompuesto la brecha, y con ello se indaga en las hipótesis de trabajo, así se ha analizado qué parte de ella se debe a diferencias de dotación de factores, y cual a deferencias de rendimiento. Las hipótesis nulas son:

$\mathrm{H}_{01}$ : Las diferencias regionales de salario se explican tanto por dotación de factores como por rendimiento de ellos.

$\mathrm{H}_{02}$ : La estructura de las brechas regionales de salarios de las mujeres es igual a la de los varones.

Pues bien, con las estimaciones se llega a distintas conclusiones; $\mathrm{H}_{01}$ se acepta para las mujeres y se rechaza para los varones y $\mathrm{H}_{02}$ se rechaza. A continuación, se analizan estos resultados considerando antecedentes de la estructura del PIB, de la ocupación y los salarios.

1. La primera hipótesis nula se verifica para las mujeres pues, efectivamente se ha calculado que la mitad de la brecha de salario (16\%) se debe a diferencias de dotación de factores, y la otra parte, a diferencias de rendimiento entre las regiones. Claramente, la menor dotación relativa en las mujeres de Magallanes tiene efectos relevantes en los salarios, este es un punto clave y plantea desafíos para la política pública, si se logran aumentar los niveles educativos superiores se podrían mejorar los ingresos laborales significativamente y reducir a la mitad la brecha con la región Metropolitana. Por otro lado, la diferencia en rendimiento se debe a la estructura ocupacional de las mujeres; ellas principalmente laboran en el sector económico de servicio, que tienen menor importancia en el PIB regional (Tabla 3), una de esas es Hoteles y Restaurantes, la que es catalogada como una actividad emergente, probablemente sea un 
sector que tiene posibilidades de ofrecer mayores retribución a los factores en la medida que mejore su competitividad.

2. En los varones se rechaza la primera hipótesis nula. La diferencia estimada es a favor de los que viven en la región de Magallanes, y se debe a que dichos factores tienen mejor rendimiento en esta región. Efectivamente, ellos trabajan en mayor proporción (que en la región Metropolitana) en la Administración Pública, Construcción (Tabla 3), sectores de gran importancia relativa en el PIB de la región (Fig. 1). Pero también en este caso se observa una diferencia negativa en dotación, y el impacto de la educación secundaria y terciaria es mayor al de las mujeres (Tabla 1 de Anexos).

3. Se rechaza la segunda hipótesis nula, esto porque las brechas salariales entre las regiones tienen estructuras diferentes. En los varones de Magallanes la diferencia es positiva aún cuando tiene menor dotación de factores que los de la región Metropolitana. La mujeres tienen menos salarios y para mejorarlo se debe aumentar la dotación e incrementar el valor agregado de los sectores emergentes en que trabajan.

Con todo, si bien se han implementado políticas públicas en Magallanes, dadas sus características de Zona Extrema, el efecto de la tendencia concentradora de la economía chilena en tono a la región Metropolitana sigue siendo dominante. Una expresión de ello son las diferencias salariales; claramente, no se han observado ventajas de salario para las trabajadoras de la región, como se ha visto, en ellas se han estimado brechas negativas.

Finalmente, los resultados aquí obtenidos podrían orientar a la política pública, en su tarea de generar mejores condiciones para la fuerza de trabajo de esta región, de manera de contrapesar las fuerzas del mercado. Claramente, para mejorar los ingresos laborales de los habitantes de Magallanes, y generar ciertas ventajas para compensar sus condiciones de Zona Extrema, la política debe orientarse de manera diferenciada. En este caso concluimos diferencias importantes por género; así, se ha observado que particularmente en mujeres se requiere mejorar las dotaciones de factores, por ejemplo en educación, en este caso está claro que el impacto se produce en niveles educativos terciarios de esta forma probablemente tendrían más posibilidades de laborar en sectores de servicio con mayor retribución, como la administración pública. Además se requiere generar mecanismos que permitan aumentar la rentabilidad en ciertos sectores que demandan trabajo femenino.

\section{BIBLIOGRAFÍA}

Aroca, P (2009). Desigualdades regionales en Chile. Foreign Affairs: Latinoamérica, 9(1), 53-62.

Atienza, M. \& Aroca, P. (2012). Concentración y crecimiento en Chile: una relación negativa ignorada (Spanish), EURE 38 (114), 257-277. dx.doi.org/10.4067/ S0250-71612012000200010

Banco Mundial (Noviembre, 2005). Chile Políticas de Excepción en Zonas Extremas Una Evaluación de Costos e Impactos y Lineamientos de Reforma. Recuperado de http:// www-wds.worldbank.org/servlet/WDSContentServer/ WDSP/IB/2005/12/20/000012009_2005122016 2013/Rendered/PDF/2735710spanish.pdf.

Beyer, H. \& Le Foulon C. (2002). Un recorrido por las desigualdades Salariales en Chile. Estudios Públicos 85 (verano), 139-175.

Biblioteca Nacional del Congreso (BCN). (2011). Política nacional para el desarrollo de localidades aisladas. BCN Minuta de Trabajo. Valparaíso, Chile.

Bravo, D., C. Sanhueza \& S. Urzúa (2008). Ability, schooling choices and gender labor market discrimination: evidence for Chile. Washington: Research Network Working paper $N^{\circ} \mathrm{R}-558$, Banco Interamericano de Desarrollo.

CIDEZE-SUBDERE (2007). Política Pública Para Territorios Especiales Aislados, Documento Base. Comité Interministerial de Zonas Extremas (CIDEZE) y Subsecretaría de Desarrollo Regional y Administrativo (SUBDERE). Recuperado de http://www.subdere. gov.cl/sites/default/files/articles-67660_recurso_2. pdf.

Correa, L. \& Soza-Amigo, S. (2012). La insoportable levedad del ser, Arica y Magallanes: La invisibilidad económica de las regiones extremas de Chile. (20062012): XIV Reunión de Economía Mundial. MS. Recuperado de http://xivrem.ujaen.es/wp-content/ uploads/2011/11/86-R-025M511.pdf.

Ferrada, L. \& Zarzosa P. (2010). Disparidades Regionales en Participación Laboral Femenina en Chile. Cuadernos de Economía, 47(136), 249-272. http://dx.doi. org/10.4067/S0717-68212010000200005.

Heckman, J. (1979). Sample selection bias as a specification 
error. Econometrica, 47(1), 140-161.

Latorre, A. (1990). Análisis Económico Espacial de la XII Región de Magallanes y Antártica Chilena. Tesis de Maestría, Universidad Austral de Chile, Valdivia.

Martinic, M. (2001). La actividad industrial en Magallanes entre 1890 y mediados del siglo XX. Historia (Santiago), 34, 91-115. http://dx.doi.org/10.4067/S071771942001003400004.

Ministerio de Desarrollo Social, Casen. Cuadros Estadísticos 2009. Observatorio Social. Recuperado de http:// observatorio.ministeriodesarrollosocial.gob.cl/casen/ casen_obj.php.

Ñopo, H. (Mayo, 2006). The Gender Wage Gap in Chile 19922003 from a Matching Comparisons Perspective. Inter-American Development Bank (BID), Research Department Working Paper, 562.

Oaxaca, R. (1973). Male - female wage diferentiales in urban labor market. International Economic Review 14 (3), 693-709.

PNUD. (2004). Desarrollo Humano en Chile, El poder: ¿para qué y para quién? Programa de las Naciones Unidas para el Desarrollo. Recuperado de http://www.
desarrollohumano.cl/pdf/2004/IDH-2004.pdf.

Riffo, L. (1999). Crecimiento y disparidades regionales en Chile: Una visión de largo plazo. Estadística y Economía INE, 2, 129-152.

Slater M. (2013). Políticas Integradoras de desarrollo y seguridad en el extremo norte, Aysén y Magallanes. Recuperado de http://www.cesim.cl/Columnas_ opinion/Pol\%C3\%ADticas\%20Integradoras\%20 de\%20Desarrollo\%20y\%20Seguridad.pdf.

Soza-Amigo, S. (2008). Relaciones intersectoriales en Magallanes: En busca de su estructura económica. Magallania 36(1), 79-102. http://dx.doi. org/10.4067/S0718-22442008000100007.

Soza-Amigo, S. (2009). Análisis comparativo para la economía magallánica desde la perspectiva de la sensibilidad de coeficientes técnicos. Magallania 37(1), 133-151. http://dx.doi.org/10.4067/S071822442009000100010.

SUBDERE, MIDEPLA \& INE. (2009). Informe indice competitividad nacional. Gobierno de Chile. Recuperado de http://www.subdere.cl/sites/default/ noticiasold/articles-75995_archivo_fuente.pdf. 
ANEXO 1

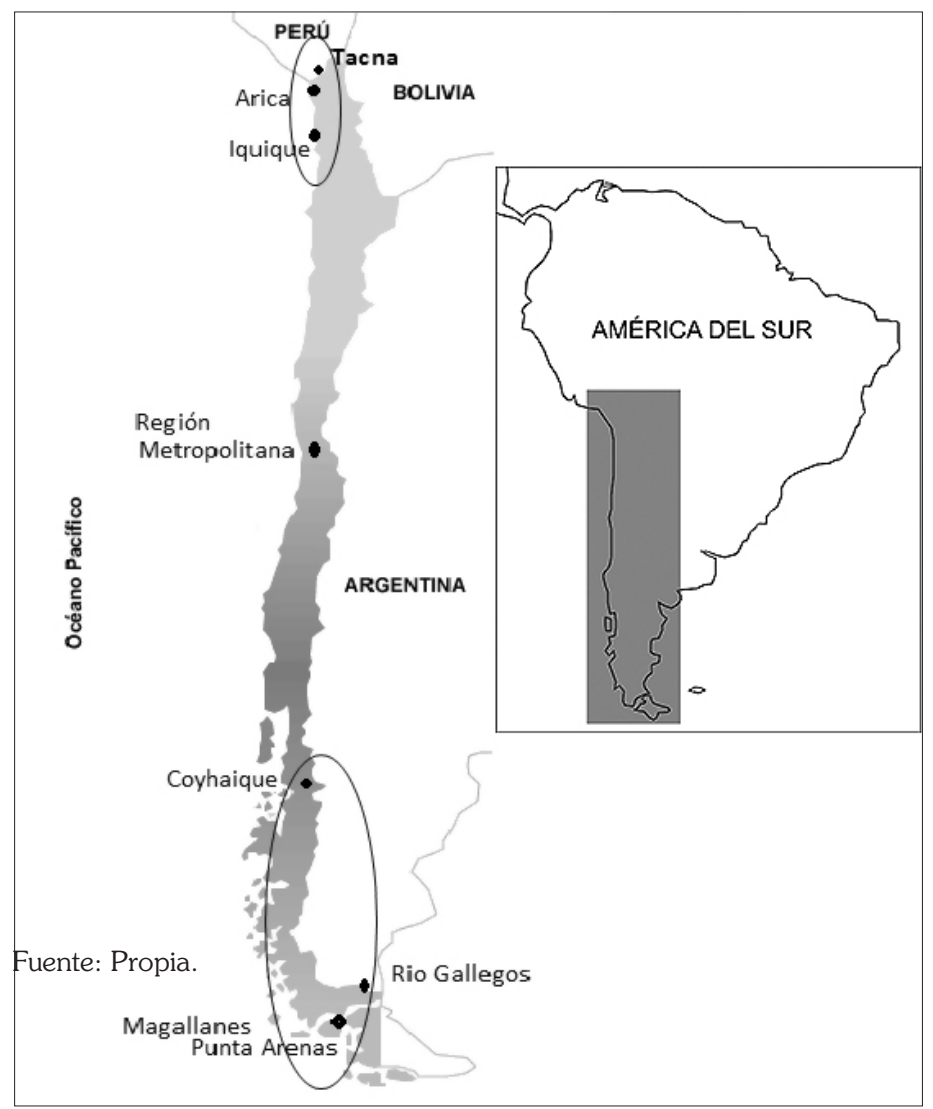

Fig.1. Chile, límites, ciudades del extremo norte y sur, y ciudades próximas más pobladas. 


\title{
ANEXO 2
}

\author{
Sesgo de Selección de la Muestra
}

Heckman (1979) trata el sesgo de selección de muestra como una variación del error de especificación, estimando el inverso del ratio de Mills obtenido de los residuos de un probit, en que se estima la probabilidad de estar ocupado mediante un probit binario, uno para cada región:

$$
P_{i}=X_{i} \alpha_{i}+v_{i}
$$

Siendo $P_{i}$, una variable dicotómica que toma el 1 si está ocupado y 0 en caso contrario. $X$ representa un vector de variables explicativas, $\alpha$ los parámetros y $v$ el término de perturbación. Con los residuos de esta estimación, se calcula el inverso ratio de Mills, posteriormente se incluye como regresor de las funciones de ingreso respectivas.
Para el cálculo probit y el ratio de Mills de este trabajo, se usa la variable dependiente condición de actividad laboral (ocupado o desocupado/ inactivo), y el vector de variables independientes, siguiendo un trabajo anterior (Ferrada \& Zarzosa, 2010) son: nivel educativo, edad, como proxy de la experiencia, estado civil y niños menores de 6 años en el hogar, todas variables categóricas.

En este estudio se corrige por sesgo de selección, tanto la muestra de hombres como de mujeres. Finalmente, las funciones de ingreso regionales se estiman en dos etapas, primero se obtiene ratio de Mills a partir de los residuos de la estimación probit y en seguida se estima la función de ingreso. 
Tabla 1 Ecuaciones de ingreso por género y región (+)

\begin{tabular}{|c|c|c|c|c|}
\hline & \multicolumn{2}{|c|}{ Hombres } & \multicolumn{2}{|c|}{ Mujeres } \\
\hline & Magallanes & Metropolitana & Magallanes & Metropolitana \\
\hline \multirow[t]{2}{*}{ Exper } & $-0,002^{*}$ & $0,0106^{*}$ & $0,0751^{*}$ & $0,0105^{*}$ \\
\hline & $(0,0011)$ & $(0,0002)$ & $(0,0014)$ & $(0,0002)$ \\
\hline \multirow[t]{2}{*}{ exper2 } & $0,0006^{*}$ & $-0,000^{*}$ & $-0,002^{*}$ & $-0,000^{*}$ \\
\hline & $(0,0000)$ & $(0,0000)$ & $(0,0000)$ & $(0,0000)$ \\
\hline \multirow[t]{2}{*}{ personas } & $0,0220^{*}$ & $-0,028^{*}$ & $-0,015^{*}$ & $-0,025^{*}$ \\
\hline & $(0,0028)$ & $(0,0003)$ & $(0,0034)$ & $(0,0004)$ \\
\hline \multirow[t]{2}{*}{ Urbano } & $0,1857^{*}$ & $0,0353^{*}$ & $0,0603^{*}$ & $0,0660^{*}$ \\
\hline & $(0,0144)$ & $(0,0032)$ & $(0,0210)$ & $(0,0046)$ \\
\hline \multirow[t]{2}{*}{ contrato } & $0,2556^{*}$ & $0,1073^{*}$ & $-0,408^{*}$ & $0,0768^{*}$ \\
\hline & $(0,0104)$ & $(0,0015)$ & $(0,0146)$ & $(0,0016)$ \\
\hline \multirow[t]{2}{*}{ secundario } & $-0,254^{*}$ & $-0,043^{*}$ & $-0,395^{*}$ & $-0,028^{*}$ \\
\hline & $(0,0117)$ & $(0,0028)$ & $(0,0345)$ & $(0,0045)$ \\
\hline \multirow[t]{2}{*}{ terciario } & $-0,205^{*}$ & $-0,029^{*}$ & $-0,448^{*}$ & $0,0742^{*}$ \\
\hline & $(0,0104)$ & $(0,0027)$ & $(0,0256)$ & $(0,0041)$ \\
\hline \multirow[t]{2}{*}{ Público } & $0,2382^{*}$ & $0,1210^{*}$ & $0,3455^{*}$ & $0,2362^{*}$ \\
\hline & $(0,0077)$ & $(0,0018)$ & $(0,0119)$ & $(0,0018)$ \\
\hline \multirow[t]{2}{*}{ edu2 } & $0,2931^{*}$ & $0,1859^{*}$ & $-0,245^{*}$ & $0,0655^{*}$ \\
\hline & $(0,0097)$ & $(0,0018)$ & $(0,0144)$ & $(0,0024)$ \\
\hline \multirow[t]{2}{*}{ edu3 } & $0,7168^{*}$ & $1,0467^{*}$ & $0,1513^{*}$ & $0,7829^{*}$ \\
\hline & $(0,0114)$ & $(0,0021)$ & $(0,0175)$ & $(0,0026)$ \\
\hline \multirow[t]{2}{*}{ Mills } & $-0,770^{*}$ & $-0,483^{*}$ & $0,1863^{*}$ & $-0,349^{*}$ \\
\hline & $(0,0137)$ & $(0,0026)$ & $(0,0211)$ & $(0,0030)$ \\
\hline \multirow[t]{2}{*}{ Constante } & $8,5903^{*}$ & $8,5311^{*}$ & $8,9274^{*}$ & $8,5202^{*}$ \\
\hline & $(0,0254)$ & $(0,0049)$ & $(0,0419)$ & $(0,0071)$ \\
\hline Prob $>F$ & 0,0000 & 0,0000 & 0,0000 & 0,0000 \\
\hline R-squared & 0,3626 & 0,3793 & 0,3287 & 0,3541 \\
\hline
\end{tabular}

"significativo al $5 \%$.

Fuente: Propia

(+)La variable dependiente es lnw. Las estimaciones de ingreso, tienen como referencia a un individuo que vive en el sector rural, sin contrato, que trabaja en el sector productivo primario, vinculado al sector público y su último curso en la educación formal es primario. 
Tabla 2 Componentes de la descomposición de salarios para hombres por Regiones

\begin{tabular}{|l|cccc|}
\hline & \multicolumn{2}{|c}{ Hombres } & \multicolumn{2}{c|}{ Mujeres } \\
Exper & Explicada & No Explicada & Explicada & No Explicada \\
exper2 & 10.186 & 0,4584 & 0,9997 & 33.458 \\
& $(0,0146)$ & $(0,1404)$ & $(0,0220)$ & -17.793 \\
personas & 0,9921 & 17.144 & 0,9978 & 0,3893 \\
& $(0,0096)$ & $(0,3031)$ & $(0,0241)$ & $(0,1524)$ \\
Urbano & 10.157 & 12.101 & 10.045 & 10.420 \\
& $(0,0048)$ & $(0,1762)$ & $(0,0049)$ & $(0,2319)$ \\
contrato & 0,9994 & 11.532 & 0,9980 & 0,9950 \\
& $(0,0008)$ & $(0,1481)$ & $(0,0017)$ & $(0,1723)$ \\
secundario & 10.045 & 11.386 & 10.063 & 0,6580 \\
& $(0,0044)$ & $(0,1266)$ & $(0,0046)$ & $(0,2105)$ \\
terciario & 10.034 & 0,9517 & 10.021 & 0,9866 \\
& $(0,0033)$ & $(0,0337)$ & $(0,0034)$ & $(0,0109)$ \\
privado & 10.003 & 0,8935 & 10.026 & 0,6171 \\
& $(0,0019)$ & $(0,0701)$ & $(0,0030)$ & $(0,1127)$ \\
edu2 & 10.216 & 10.322 & 10.231 & 10.278 \\
& $(0,0095)$ & $(0,0342)$ & $(0,0139)$ & $(0,0375)$ \\
edu3 & 10.126 & 10.643 & 10.027 & 0,8515 \\
& $(0,0100)$ & $(0,0710)$ & $(0,0042)$ & $(0,1250)$ \\
Mills & 0,9035 & 0,9242 & 0,8932 & 0,8456 \\
& $(0,0412)$ & $(0,0336)$ & $(0,0346)$ & $(0,0775)$ \\
Constante & 10.104 & 0,9057 & 0,9865 & 15.064 \\
& $(0,0165)$ & $(0,0503)$ & $(0,0099)$ & $(0,3528)$ \\
\hline Observaciones & 10.612 & & 15.239 \\
& & $(0,3202)$ & & $(0,8970)$ \\
\hline
\end{tabular}

Fuente: Propia

Tabla 3 Estimación de Brecha mediante descomposición Blinder-Oaxaca

Brecha para Hombres, número de observaciones $=9068$

\begin{tabular}{|l|c|c|c|c|c|}
\hline Lw & $\exp (\mathrm{b})$ & $\begin{array}{c}\text { Desviación } \\
\text { estándar }\end{array}$ & $\mathrm{P}>|\mathrm{z}|$ & \multicolumn{2}{c|}{ Intervalo de Confianza al 95\% } \\
Predicción_R, Magallanes & $7462,144^{*}$ & 4.069 .584 & 0,000 & 6.705 .669 & 8.303 .957 \\
Predicción R, Metropolitana & $7372,982^{*}$ & 1.123 .933 & 0,000 & 7.155 .953 & 7.596 .593 \\
Diferencia & 1.012 .093 &, 0573116 & 0,832 &, 9057735 & 1.130 .892
\end{tabular}

Brecha para Mujeres, número de observaciones $=5981$

\begin{tabular}{|l|c|c|c|c|c|}
\hline Lw & exp(b) & $\begin{array}{c}\text { Desviación } \\
\text { estándar }\end{array}$ & P> $|z|$ & \multicolumn{2}{|c|}{ Intervalo de Confianza al 95\% } \\
\hline Predicción_R, Magallanes & $5584,309^{*}$ & 4.984 .613 & 0,000 & 4.688 .029 & 6.651 .944 \\
\hline Predicción R, Metropolitana & $6650,019^{*}$ & 1.069 .857 & 0,000 & 6.443 .602 & 6.863 .048 \\
\hline Diferencia &, $8397432^{* *}$ &, 0761641 & 0,054 &, 7029803 & 1.003 .113 \\
\hline
\end{tabular}

*significativo al 5\%; ** significativo al $10 \%$.

Fuente: Propia 
